\title{
A STRAIN ENERGY DENSITY THEORY FOR MIXED MODE CRACK PROPAGATION IN RUBBER-LIKE MATERIALS
}

\author{
AbDelkader Boulenouar \\ Djillali Liabes University of Sidi Bel-Abbes, Mechanical Engineering Department, Laboratory of Materials and Reactive \\ Systems, Larbi Ben Mhidi, Algeria; e-mail: aek_boulenouar@yahoo.fr \\ Noureddine BenseddiQ \\ Mechanics Laboratory of Lille, CNRS UMR 8107, Ecole Polytech'Lille, University of Lille, France \\ Mohamed Merzoug, Nabil Benamara, Mohamed Mazari \\ Djillali Liabes University of Sidi Bel-Abbes, Mechanics and Physics of Materials Laboratory, Larbi Ben Mhidi, Algeria
}

\begin{abstract}
In this paper, a numerical modeling of crack propagation for rubber-like materials is presented. This technique aims at simulating the crack growth under mixed-mode loading based on the strain energy density approach. At each crack increment length, the kinking angle is evaluated as a function of the minimum strain energy density (MSED) around the crack tip, using the Ansys Parametric Design Language (APDL). In this work, numerical examples are illustrated to demonstrate the effectiveness, robustness and accuracy of the computational algorithm to predict the crack propagation path. The results obtained show that the plan of crack propagation is perpendicular to the direction of the maximum principal stretch. Moreover, in the framework of linear elastic fracture mechanics (LEFM), the minimum values of the density are reached at the points corresponding to the crack propagation direction.
\end{abstract}

Keywords: strain energy density, mixed mode, hyper-elastic, crack propagation

\section{Introduction}

Today rubbers are used in many engineering industries. The demand for such materials and their performance is continually increasing. The performance is limited, among other factors, by the initiation of cracks. Hence, it is necessary to develop procedures that allow an exact determination of a fracture criterion of these materials. To study fracture rubbers we can use two approaches: the global approach and the local approach. The global approach of the fracture mechanics, originated from Griffith $(1920,1924)$, represents a good method allowing the characterisation of the materials fracture. It assumes that initiation and growth of cracks begin from pre-existing defects in the structure. In the '50s, Rivlin and Thomas (1953) generalized the Griffith's energy approach and defined the concept of tearing energy $T$ as the energy released per unit area of crack surface growth. The parameter $T$ can be calculated analytically from a few specimen geometries proposed by several authors (Rivlin et Thomas, 1953; Greensmith, 1963; Andrews, 1974).

The global energy approach is used qualitatively to characterize the rupture of materials under simple loading (mode I); it is not adapted for complex loadings (mode I+II). In this case, the quantitative local approach represents a good alternative which makes it possible to indicate the crack initiation and direction.

In linear elastic fracture mechanics, the various fracture criteria for cracks subjected to mixed mode loading have been introduced for the determination of the propagation direction and the critical stress such as the maximum tangential stress criterion (Erdogan and Sih, 1963; Chang, 1981; Maiti and Smith, 1983), maximum principal tangential stress criterion (Maiti et Smith, 
1983), maximum strain criterion (Wu, 1974; Chang, 1981), and strain energy density criterion (Sih, 1974; Theocaris, 1984). Almost all these criteria postulate that crack initiation occurs at the crack tip and propagate towards the radial direction.

Sih's (Sih, 1973, 1974; Sih and Macdonald, 1974; Kipp and Sih, 1975) strain energy density criterion (SEDC) assumes the strain energy density to be investigated for an element around the crack tip as the starting point and the strain energy density factor as a basic parameter. It is then postulated that crack initiation will occur at the crack tip in the radial direction along which the strain energy density factor $S$ is minimum, and the crack will begin to propagate when the factor $S$ reaches some critical value.

The strain energy density criterion was validated experimentally and numerically on fragile materials (Theocaris, 1984; Boulenouar et al., 2016, 2013a; Benouis et al., 2015; Ayatollahi and Sedighiani, 2012; Pegorin et al., 2012; Choi et al., 2006) and ductile materials (Komori, 2005; Chow and Xu, 1985; Carpinteri, 1984). In the framework of large deformations, this criterion was used by Hamdi et al. (2007) on filled rubber materials to predict the initial crack orientation of a central crack.

The objective of this paper is to present the numerical modeling of the crack propagation trajectory for rubber-like materials. Using the Ansys Parametric Design Language (APDL) (2006), the crack direction is evaluated as a function of the minimum strain energy density $(d W / d V)_{\min }$ around the crack tip. The numerical examples are included to illustrate the validation of the numerical approach for crack growth simulation in hyper-elastic materials.

\section{The strain energy density theory}

The SED fracture criterion locally focuses on the continuum element ahead of the crack and is based on the notion of weakness or severity experienced by the local material. Failure occurs when the critical amount of strain energy dW is accumulated within the element volume $d V$ and the crack is then advanced incrementally in the corresponding direction (Sih, 1974; Sih and Barthelemy, 1980). The strain energy density function $d W / d V$ is assumed to have the following form

$$
\frac{d W}{d V}=\frac{S}{r}
$$

where $S$ is the strain energy density factor and $r$ is the distance from the crack tip. The minimum of the strain energy density factor $S_{\min }$ around the crack tip determines the likely direction of crack propagation.

The strain energy density can be determined directly from the relationship

$$
\frac{d W}{d V}=\frac{1}{2} \sigma^{T} \varepsilon
$$

The computed discrete values for $S$ are then fitted with an approximation function which enables simple determination of the local minimum. The strain density function has several local minimuma around the crack tip, where the global minimum is not necessarily the true solution, as it can be observed in Fig. 1.

The physically meaningful minimum of the strain density function $S_{\min }$ can be found numerically by incremental search for a local minimum in possible crack extension directions $\theta_{i}$ in the range $\pm \pi$ around the crack tip (Fajdiga et al., 2007).

Fajdiga et al. (2007) used the position of integration points to define the corresponding angle of calculated strain energy density and strain energy density factor $S$ around the crack tip. In this study, the minimum strain energy density $(d W / d V)_{\min }$ is computed by introducing a ring of elements around the crack tip. At each crack increment, the crack direction is evaluated as a function of the angle between the centre of the element and the crack axis (Fig. 2). 


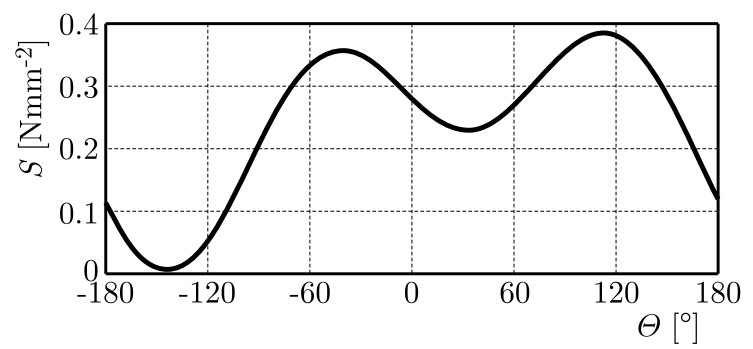

Fig. 1. Strain energy density factor $S$ as a function of the angle $\theta$

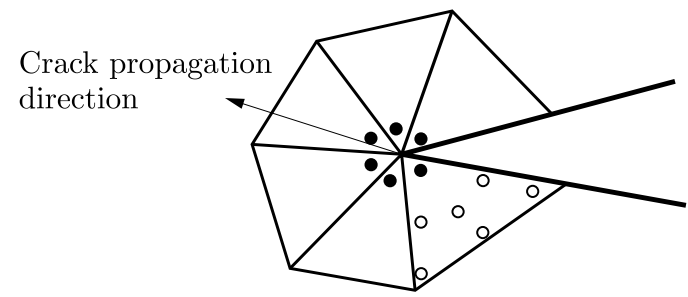

Fig. 2. Direction of propagation using the minimum strain energy density

\subsection{Modeling of the hyperelasticity}

The mechanical behavior of rubber materials is generally described using a hyper-elastic formalism defined by a function $d W / d V$ called the strain energy density. The establishment of the law of this behavior is a necessary step to perform analytical and/or numerical calculations to develop or exploit multiaxial tests. These behavior laws should be specific to the strain ultimate levels and should allow description of multiaxial loadings accurately.

In this study, we based on the experimental data of Hamdi et al. (2006), Hamdi (2006) using four tests: uniaxial tension (UT), uniaxial compression (UC), pure shear (PS) and equibiaxial tension (ET). The tests were performed on two rubber materials: natural rubber and styrene butadiene rubber.

Based on these tests, the rubber materials are modeled by a hyperelastic potential developed for quasi-incompressible rubbers using Yeoh's model (Yeoh, 1990), Eq. (2.3) ${ }_{1}$, for natural rubber NR and Ogden's model (Ogden, 1984), Eq. $(2.3)_{2}$, for Styrene Butadiene rubber SBR

$$
\begin{aligned}
& \frac{d W}{d V}=C_{10}\left(I_{1}-3\right)+C_{20}\left(I_{1}-3\right)^{2}+C_{30}\left(I_{1}-3\right)^{3} \\
& \frac{d W}{d V}=\sum_{k=1}^{N} \frac{\mu_{k}}{\alpha_{k}}\left(\lambda_{1}^{\alpha \kappa}+\lambda_{2}^{\alpha \kappa}+\lambda_{3}^{\alpha \kappa}-3\right)
\end{aligned}
$$

where $d W / d V$ is strain energy density, $C_{10}, C_{20}, C_{30}, \mu_{k}, \alpha_{k}$ are material parameters to be determined, $\lambda_{1}, \lambda_{2}$ and $\lambda_{3}$ are principal extensions.

The hyper-elastic coefficients of the studied materials are given in Table 1.

Table 1. Values of the material parameters values (Hamdi et al., 2006; Hamdi, 2006)

\begin{tabular}{|c|c|c|c|c|}
\hline Materials & \multicolumn{4}{|c|}{ Material parameters } \\
\hline \hline \multirow{2}{*}{$\mathrm{NR}$} & $C_{10}[\mathrm{MPa}]$ & $C_{20}[\mathrm{MPa}]$ & $C_{30}[\mathrm{MPa}]$ & \\
\cline { 2 - 5 } & 0.298 & 0.014 & 0.00016 & \\
\hline \multirow{2}{*}{ SBR } & $\mu_{1}[\mathrm{MPa}]$ & $\alpha_{1}$ & $\mu_{2}[\mathrm{MPa}]$ & $\alpha_{2}$ \\
\cline { 2 - 5 } & 0.638 & 3.03 & -0.025 & -2.35 \\
\hline
\end{tabular}


The relevance of these material models was evaluated by Hamdi (Figs. 3a and 3b) for different loading (UT, UC, ET and PS), comparing the calculated stress-strain analytically with those obtained from the experiment.
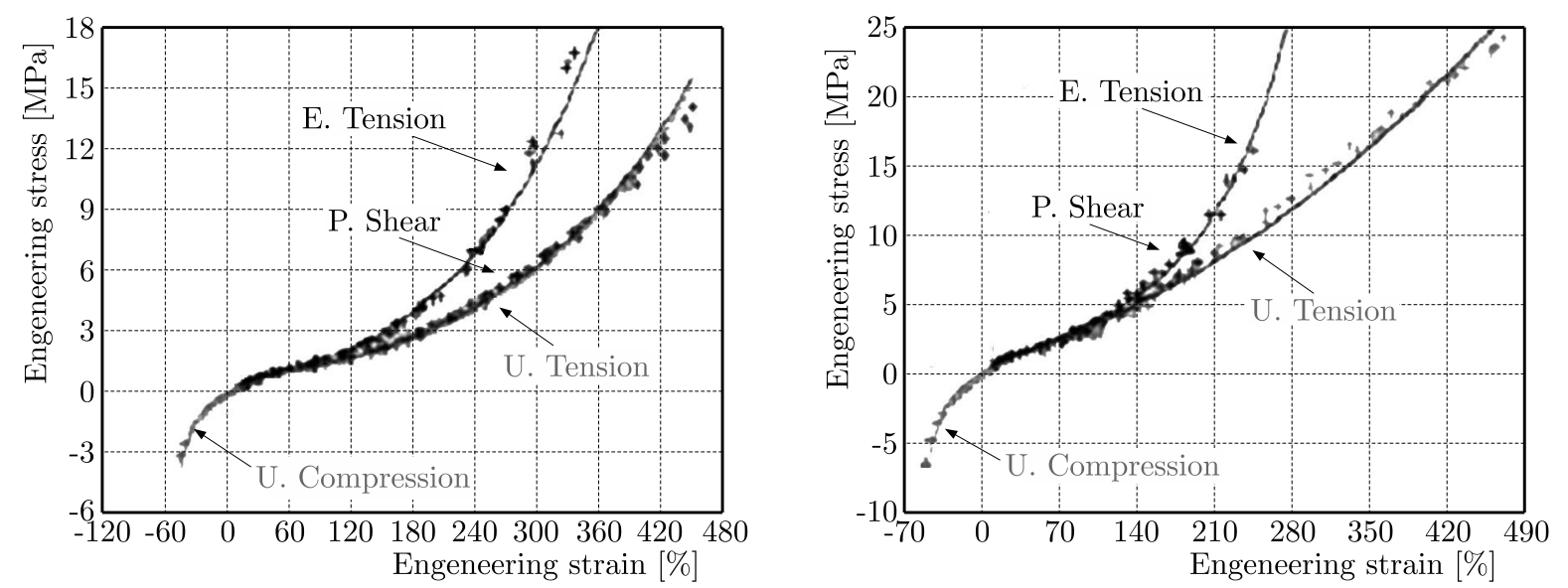

Fig. 3. Engineering stress-strain evolution for NR (left) and SBR (right) (Hamdi et al., 2006; Hamdi, 2006; Boulenouar and Mazari, 2009)

\subsection{Finite element modeling and crack growth algorithm}

This Section presents a finite element analysis for the modeling of fracture problems in rubber-like materials using the remeshing technique. Figure 4 shows a flow-chart of the prepared

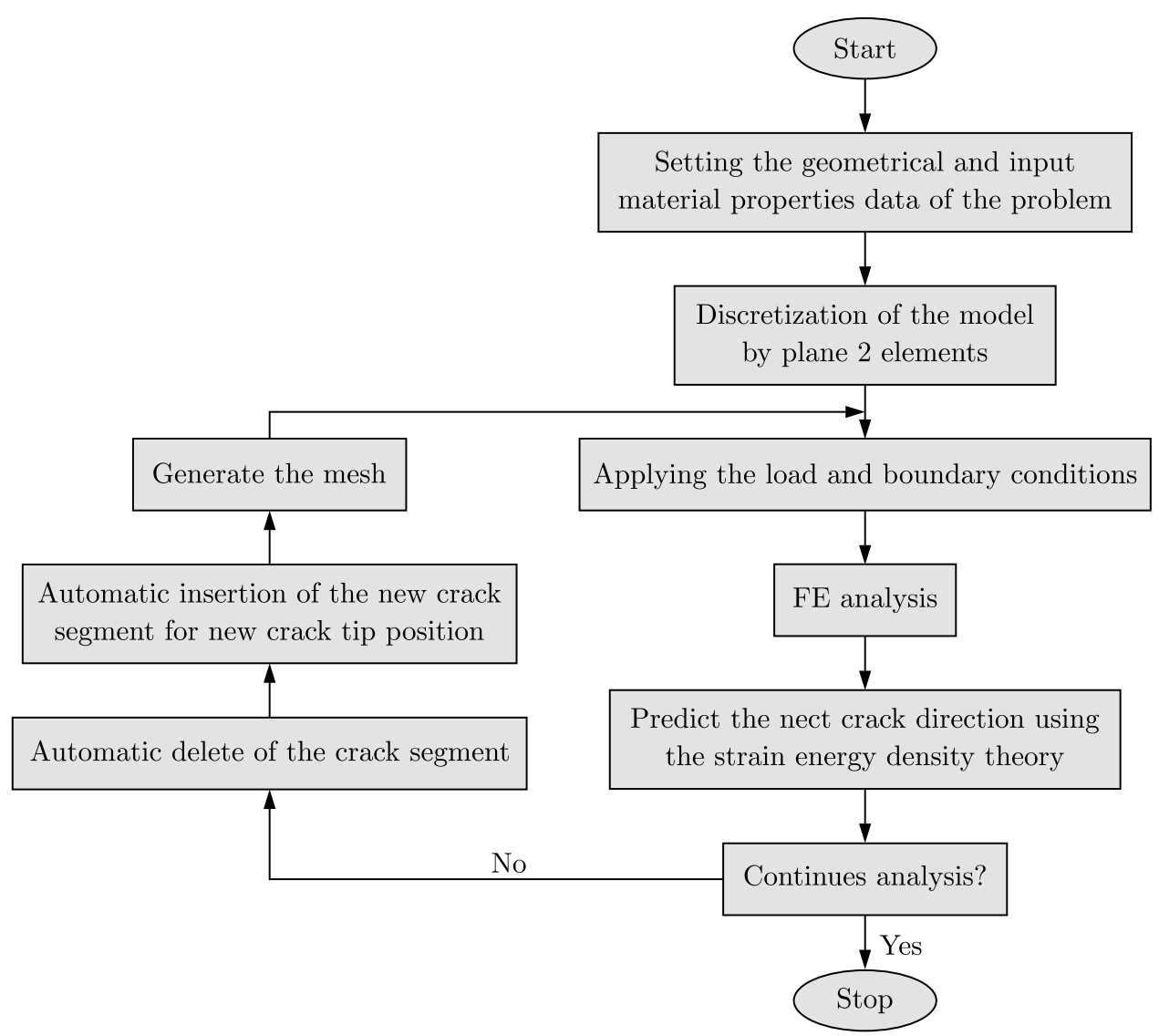

Fig. 4. Flow chart of the FEM algorithm of the problem 
APDL code based on a combination of the finite element analysis and the strain energy density concept. According to the algorithm, after initial geometrical and physical modeling of the problem, the mesh pattern is generated around crack tip. In order to find a new crack tip position at each step of propagation, the Strain Energy Sih's Theory is employed to obtain the kinking angle $\theta_{0}$ as a function of the minimum strain energy density $(d W / d V)_{\min }$. At each increment $\Delta a$ of crack propagation, the special mesh is generated around crack tip, using the quadratic six-node triangular element. It is noted that the same numeration of the nodes around the crack tip is taken during the crack propagation to evaluate automatically the new crack tip position. The algorithm is repeated until ultimate failure of the material or by using another criterion for termination of the simulation process. Figure 5 illustrates the crack propagation mechanism proposed in this study.

(a)

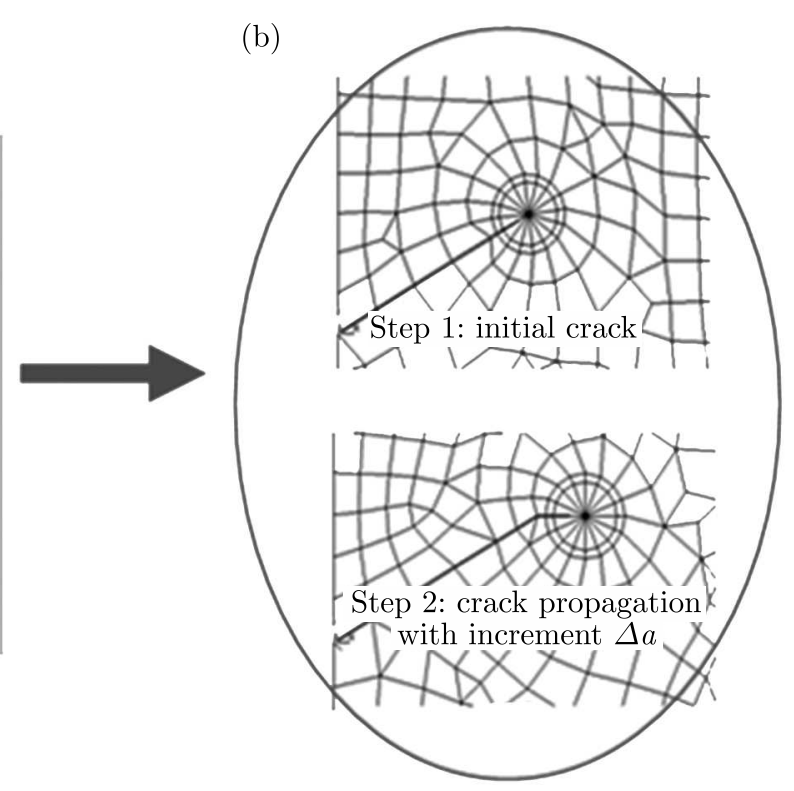

Fig. 5. (a) Crack propagation mechanism proposed in this study, (b) FE modeling for crack propagation

\section{Results and discussion}

\subsection{Minimal strain energy density criterion}

The geometry of the single edge cracked plate $(100 \mathrm{~mm} \times 50 \mathrm{~mm})$ with an initial crack ( $a=30 \mathrm{~mm})$ is considered for 2-dimensional finite element analysis. The pre-existing crack is inclined to the horizontal axis with the angle $\alpha$ (Fig. 6). The FE calculation has been achieved by gradually increasing the elongations $\lambda_{1}$ applied to the nodes located at the top of the plate.

For mesh generation of the cracked plate, the element type PLANE183' of ANSYS code is used, as shown in Fig. 7a. It is a higher order two dimensional, 8-node element having two degrees of freedom at each node (translations in the nodal $x$ and $y$ directions), quadratic displacement behavior and the capability of forming a triangular-shaped element, which is required at the crack tip areas.

Due to singular nature of the stress field in the vicinity of the crack, the singular elements, shown in Fig. 7b, are considered at each crack tip area, which is modeled with a finer mesh. Plane stress and large strains are assumed in the analysis.

The rings of the elements surrounding the tip of the crack are employed. This mesh will be used to determine the strain energy density in these elements in order to determine the kinking 


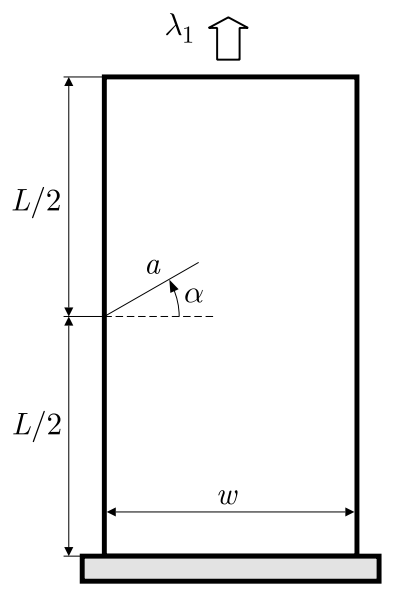

Fig. 6. Geometry of the single edge cracked plate

(a)

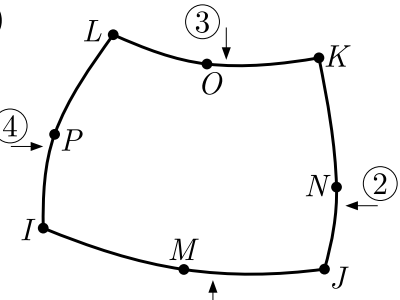

(1) (b)

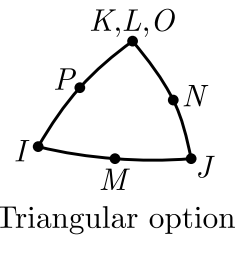

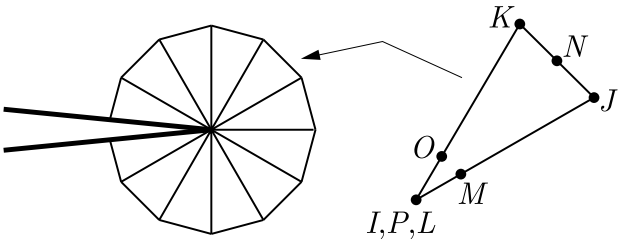

Fig. 7. (a) PLANE183 eight-node finite element and (b) singular option

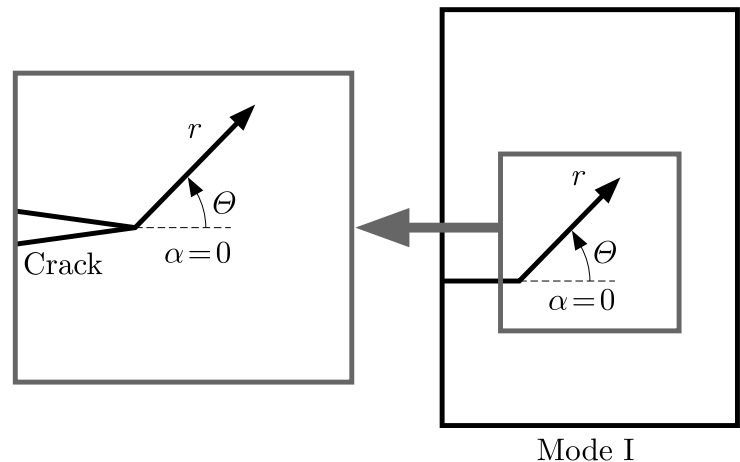

Mode I

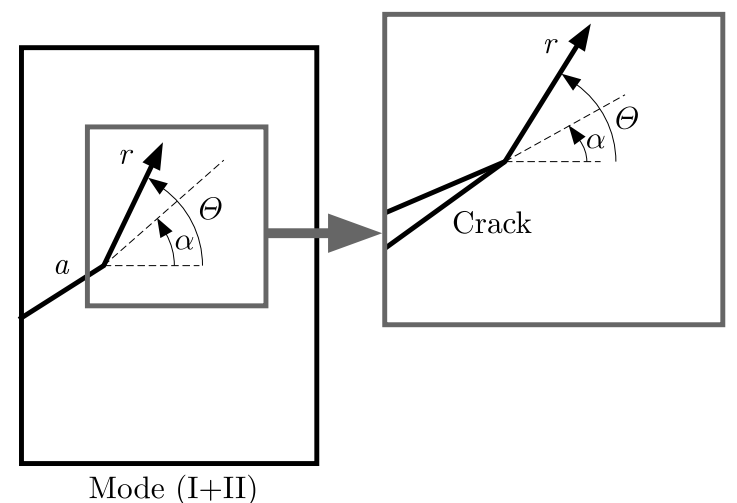

Mode $(\mathrm{I}+\mathrm{II})$

Fig. 8. Description of the parameters $r, \alpha$ and $\theta$

angle $\theta$ in the direction for which this energy density is minimal $(d W / d V)_{\min }$. Figure 8 illustrates description of the parameters $r, \alpha$ and $\theta$, where $r$ is the distance from the crack-tip, $\alpha$ is the crack inclination angle and $\theta$ is the kinking angle.

The specifications of the crack tip mesh and a close up view for the crack inclination angle $\alpha=-30^{\circ}$ are shown in Fig. 9. In this figure, $r_{i}$ represents the distance between the crack tip and the center of the element $c$.

For the two materials studied NR and SBR, Figs. 10 show the evolution of the strain energy density $d W / d V$ as a function of the angle $\theta$. The results obtained are traced for several values of the radius $r_{i}(1.25,1.75,2.5$ and $3 \mathrm{~mm})$ and for three orientations of the initial crack $\alpha=30^{\circ}$, $0^{\circ}$ and $-30^{\circ}$. These figures highlights that out of the core region surrounding the crack tip (in our case $r \leqslant r_{1}$ ), the minimum of $d W / d V$ is reached for a constant value independently of the distance $r$. The angle $\theta_{0}$ corresponds to the horizontal direction perpendicular to the loading. It is noted that the observations obtained are similar to those obtained by Hamdi (2006). 

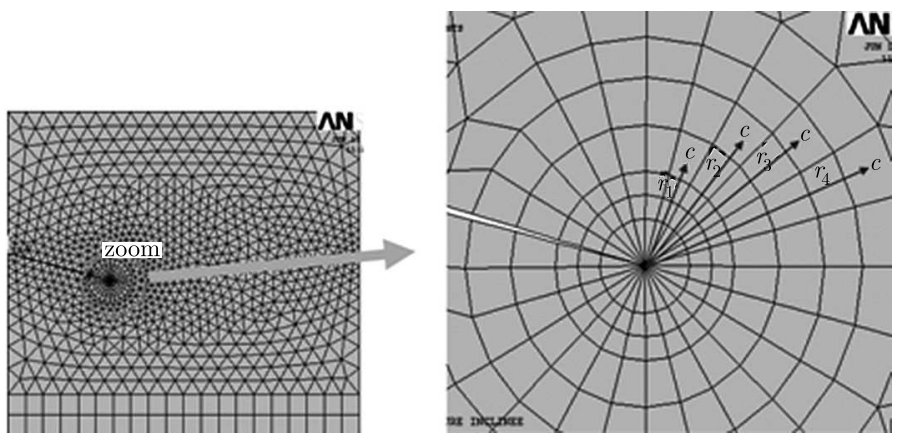

Fig. 9. The ring of elements around the crack tip

Figure 11a illustrates an example of the application of the SED approach in the case of rubbers NR and SBR under mixed-mode loading. This figure shows the evolution of the parameter $S$, called the strain-energy-density factor (with $S=(d W / d V) r$ ). The results obtained are given vs. the radius $r_{i}$ and the polar angle $\theta$ for an inclination angle $\alpha=30^{\circ}$. The curves plotted in Fig. 11a show that out of the core region surrounding the crack-tip, the minimum of the parameter $S$ is reached for a constant value independently of the radius $r$.

The angle equalizes here to $-30^{\circ}$, corresponding contrary to the value of the initial orientation of the crack $(-\alpha)$ calculated for the reference mark $O x^{\prime} y^{\prime}$ (Fig. 11b). These results show a good agreement with the results obtained by Hamdi et al. (2007) for other styrene butadiene rubber filled with carbon black SBR2 (Fig. 12).

\subsection{Effect of the number of elements surrounding the crack tip}

For the strain energy density criterion, the precision is strongly related to the number of elements surrounding crack tip zone. For this purpose, we examined the influence of the mesh size (or number of the elements) on the strain energy density variation. For that, the density $d W / d V$ is evaluated for different numbers of elements surrounding the crack tip. In this study, the numbers of elements considered are 16, 40 and 56 (Fig. 13).

For different numbers of elements surrounding the crack tip zone and for several values of the radius $r$, Figs. 14 and 15 illustrate respectively for NR and SBR the evolution of the strain energy density $d W / d V$ as a function of the initial angle of crack propagation $\theta$ (with $\alpha=30^{\circ}$ ). The results obtained show that the precision is related to the elements size around the crack tip.

The results obtained are similar to those found in the case of a plate of rubbers (NR and SBR) containing a central crack (Hamdi, 2006) (Fig. 16). It is clear that this crack is propagated according to the horizontal direction perpendicular to the loading direction and independently of the inclination angle $\alpha$. Thus, the crack propagation direction is null, according to the reference mark of calculation $(O x y)$.

To better show the influence of the elements size around the crack tip on the determination of the initial angle of crack propagation $\theta_{0}$, Fig. 17 illustrates the variation of the angle $\theta_{0}$ as a function of $(d W / d V)_{\min }$ for various numbers of elements surrounding the crack tip. The curves obtained show more elements around the crack tip make the crack direction $\theta_{0}$ at each crack increment length and the final path of crack propagation more precise.

\subsection{Crack propagation path}

In this Section, two simple examples are proposed to validate our model of the initial crack propagation in rubber materials.

Figure 18 shows four steps for the extension of the initial crack (with $\alpha_{=} 0^{\circ}$ ). As expected, the crack propagates horizontally, depending on mode I loading. The results obtained allow us 

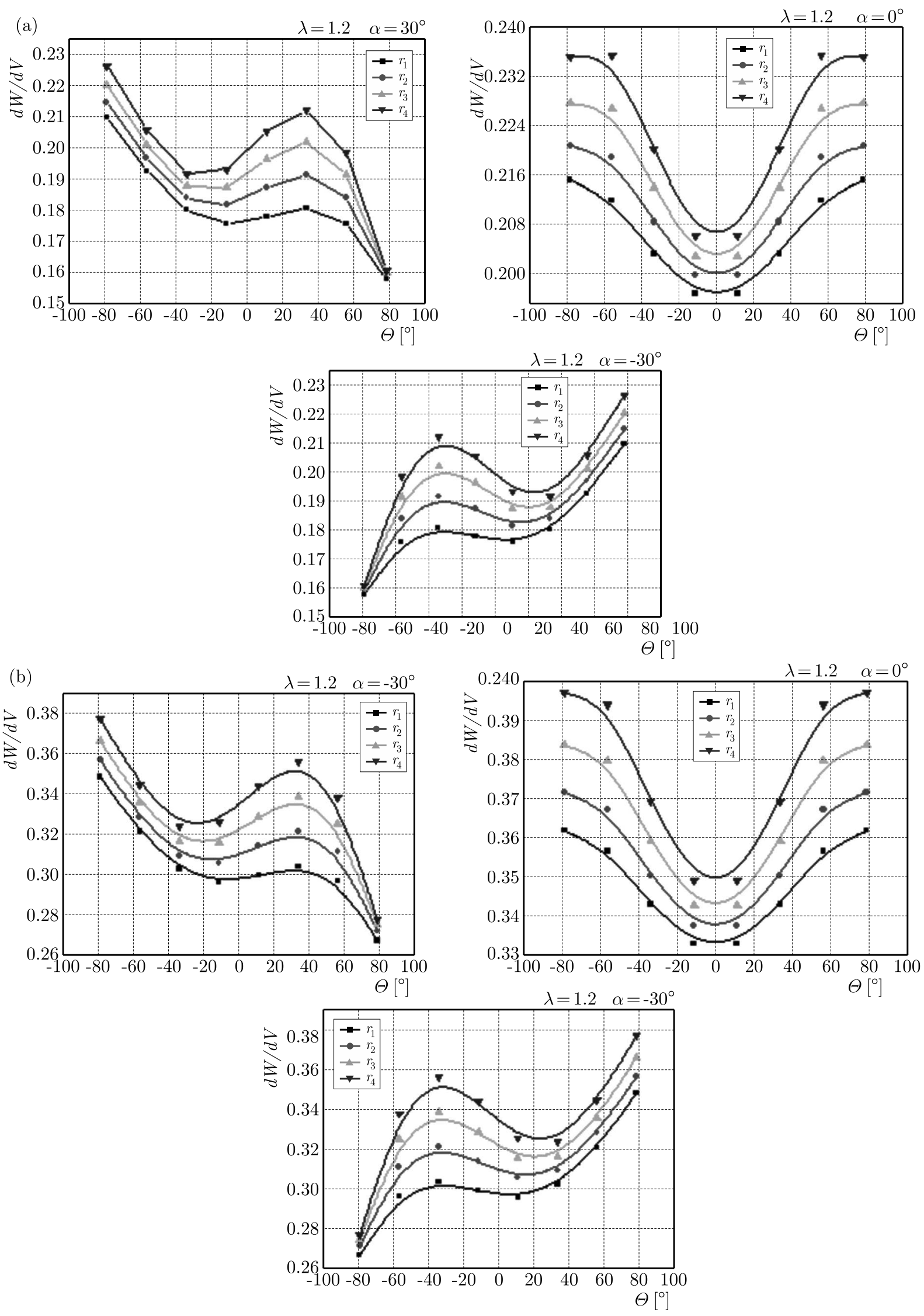

Fig. 10. Evolution of $d W / d V$ as a function of $\theta$ for several values of the radius $r$ (with $\alpha=30^{\circ}, 0^{\circ}$ and $-30^{\circ}$ ); (a) NR, (b) SBR 
(a)
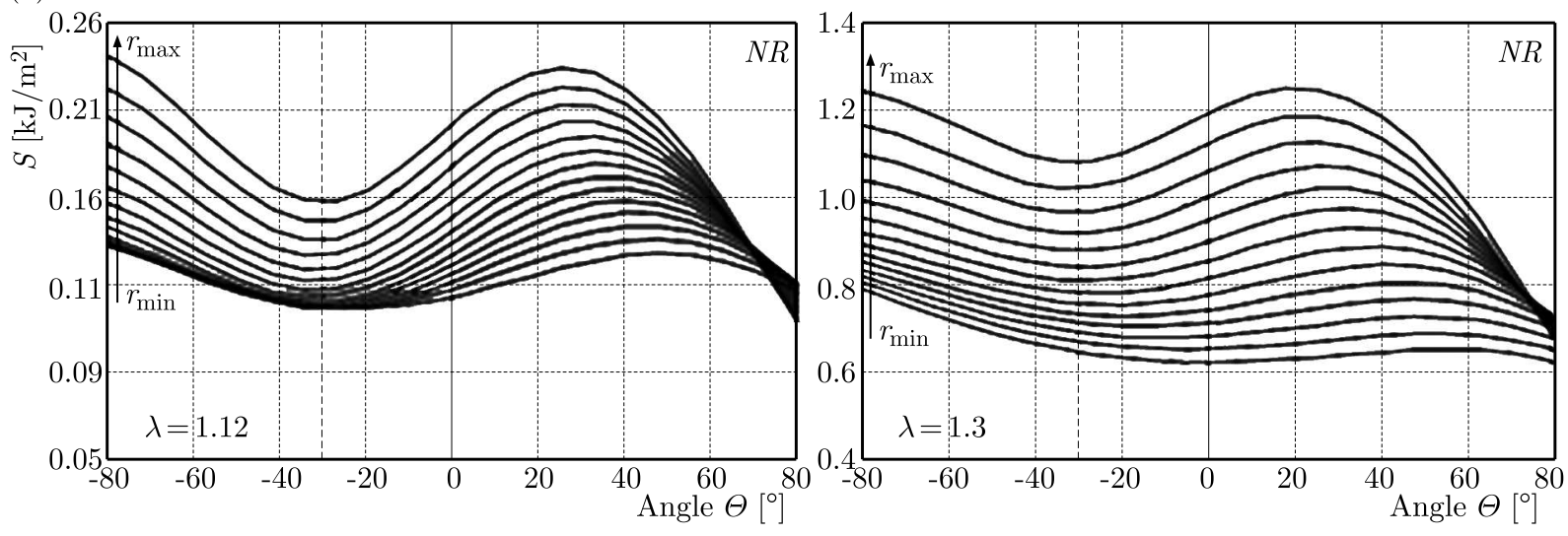

$r[\mathrm{~mm}]=\{1.14,1.47,1.84,2.25,2.70,3.21,3.78,4.41,5.11,5.89,6.76,7.72,8.80,10.00\}$
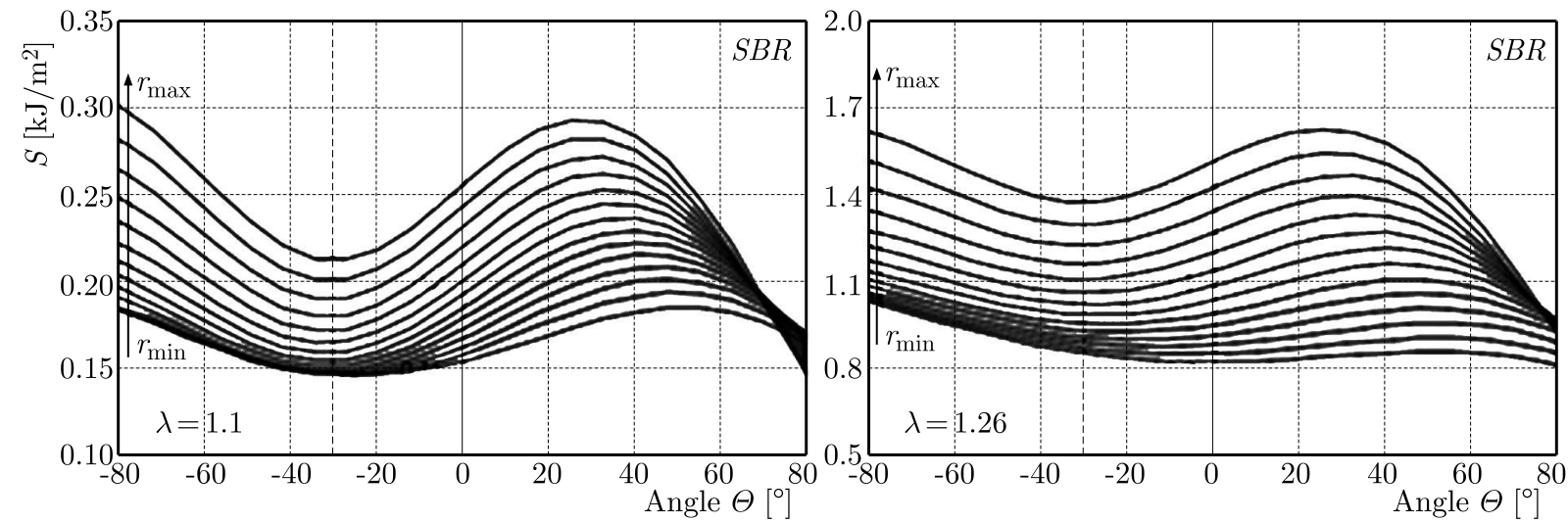

(b)

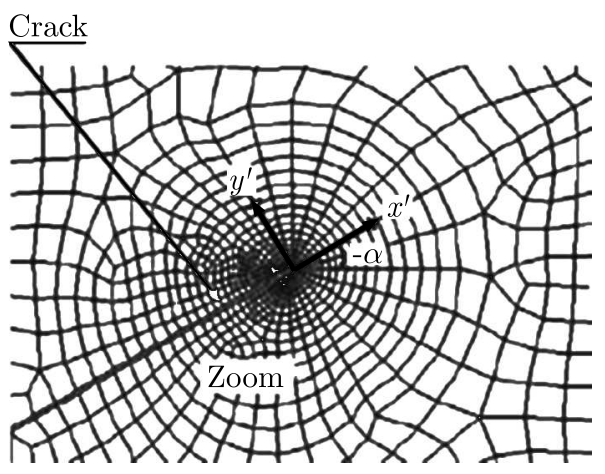

Fig. 11. (a) Evolution of $S$ vs. $\theta$ and $r$ for $\alpha=30^{\circ}$ (for NR and SBR), (b) elements around the crack tip
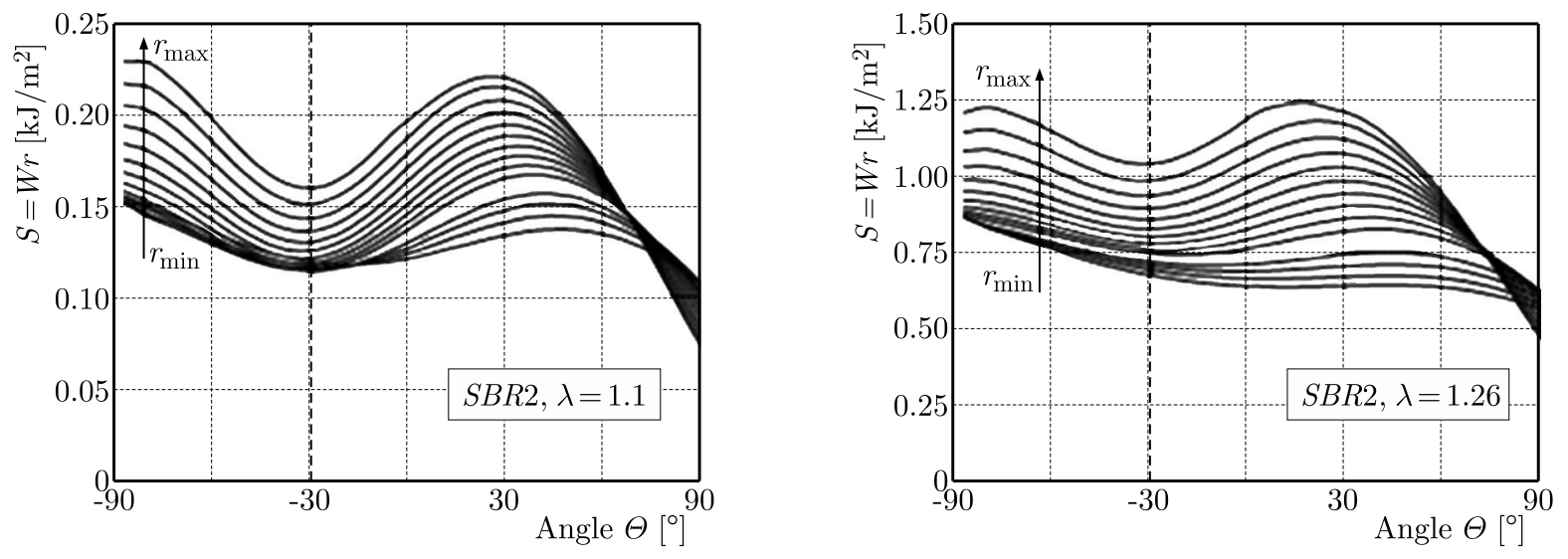

$r=\{0.85,1.14,1.47,1.84,2.25,2.70,3.21,3.78,4.41,5.11,5.89,6.76,7.72,8.80,10.00\}[\mathrm{mm}]$

Fig. 12. Evolution of $S$ as a function of $\theta$ for several values of the radius $r$ and for two given stretches $\left(\alpha=30^{\circ}\right)$ 


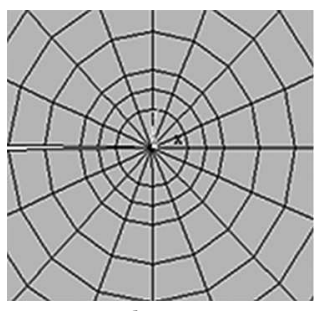

16 elements

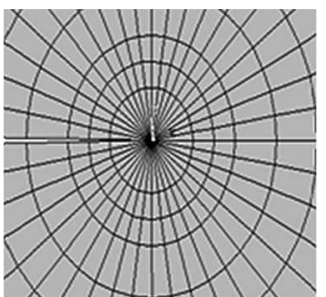

40 elements

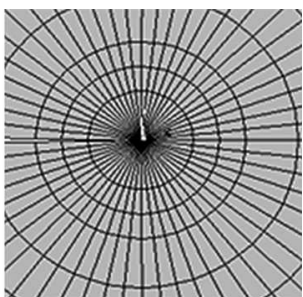

56 elements

Fig. 13. Elements sizes around the crack tip
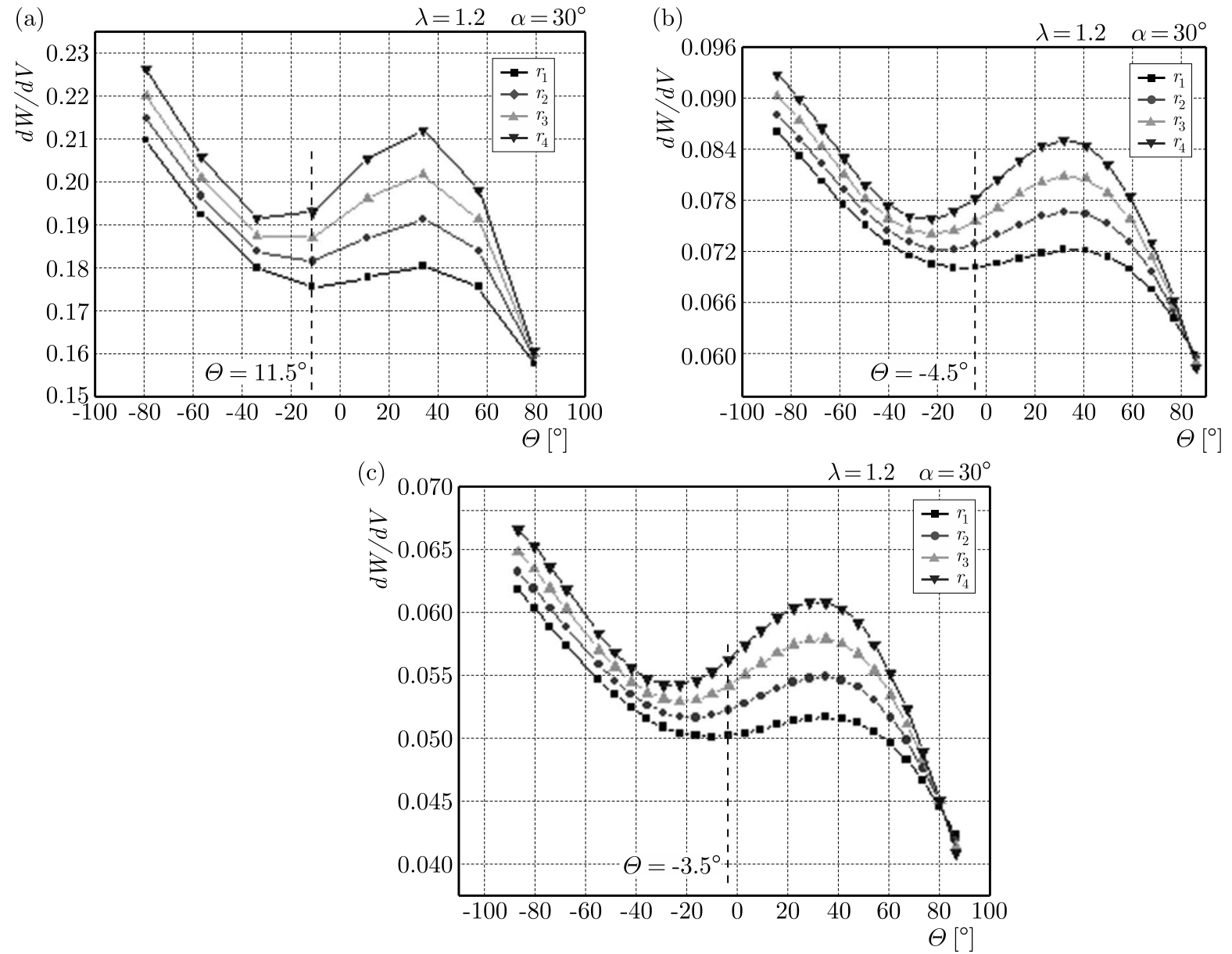

Fig. 14. Influence of the meshing on the evolution of $d W / d V\left(\mathrm{NR}, \alpha=30^{\circ}\right)$; (a) 16 elements,

(b) 40 elements, (c) 56 elements

to conclude that the criterion implemented gives a good crack path under mode I loading. This crack propagation trajectory is similar to the observed in the case of elastic linear materials using the strain energy density approach (Boulenouar et al., 2013a, 2014) or another crack propagation criterion (Boulenouar et al., 2013b; Alshoaibi and Ariffin, 2006).

In what follows, we propose to study the propagation path of a crack inclined to the horizontal axis with an angle $\alpha=30^{\circ}$. The kinked angle and the crack propagation are made under plane stress problems with the same loading conditions. Figure 19 presents four steps of the crack propagation path. As expected, the crack reorients towards the vertical loads and propagates horizontally until the end.

The same trajectory of crack propagation has been checked for another material elastomer presented by Hamdi et al. (2007), see Fig. 20. The results obtained show that this crack is pro- 

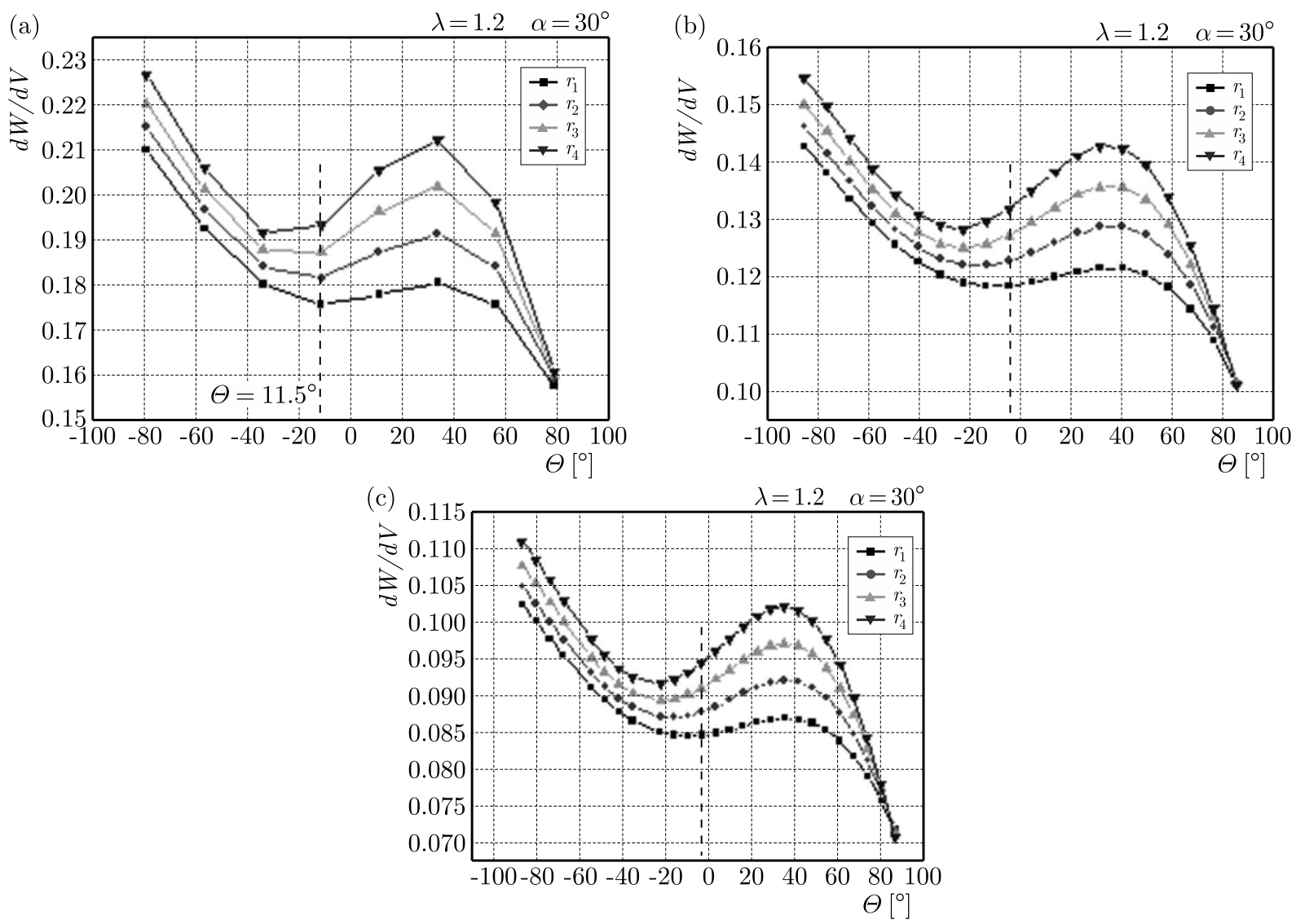

Fig. 15. Influence of the meshing on the evolution of $d W / d V\left(\mathrm{SBR}, \alpha=30^{\circ}\right)$; (a) 16 elements,

(b) 40 elements, (c) 56 elements

(a)

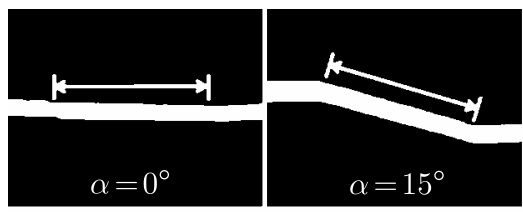

(b)

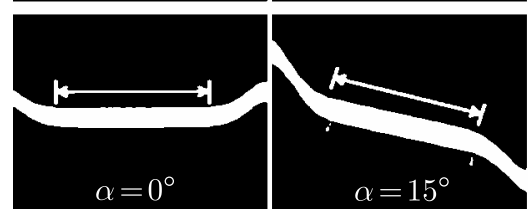

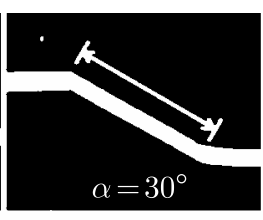
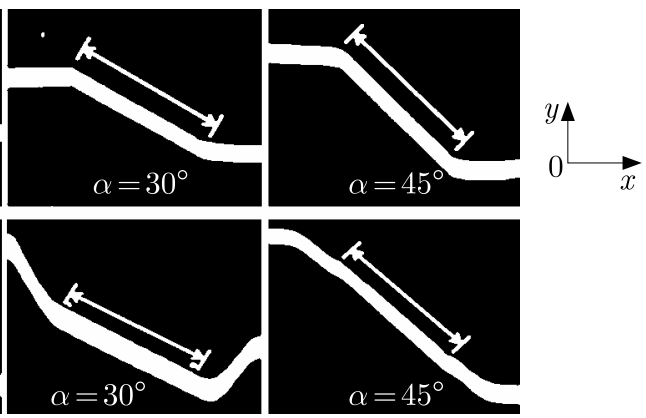

Fig. 16. Initiation and propagation of a pre-existing crack in rubbers (Hamdi, 2006)

pagated horizontally, according to mode-I (opening mode). In linear elastic fracture mechanics (LEFM), this behavior of crack propagation is similar to that observed in experiments by Bian and Taheri (2011), and numerically by Alshoaibi and Ariffin (2008).

In the last example, Legrain (2007) and Legrain et al. (2005) applied the X-FEM approach to large strain fracture mechanics. Figure 21 illustrates the crack propagation path estimated for a single edge cracked plate in rubber containing an inclusion. As waited, the crack is propagated horizontally, far from a defect. 


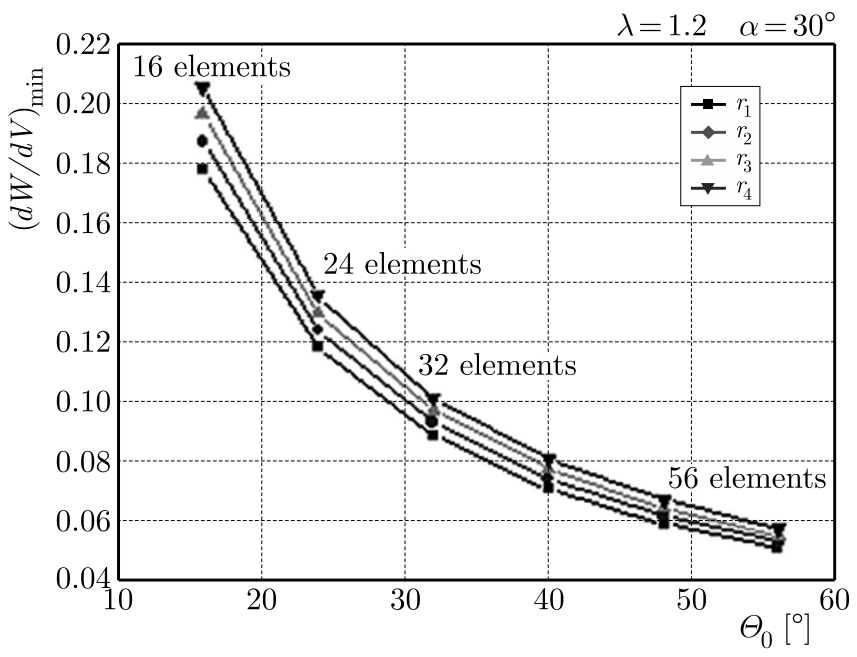

Fig. 17. Influence of the meshing on the determination of the angle $\theta_{0}\left(\mathrm{SBR}, \alpha=30^{\circ}\right)$

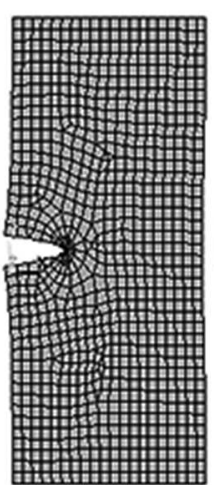

Etape 1

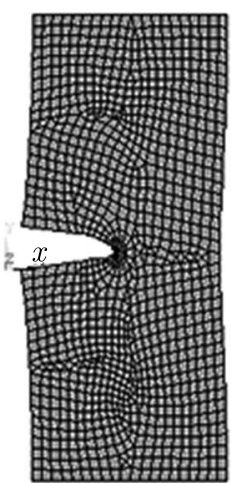

Etape 4

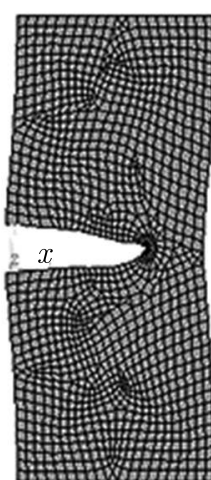

Etape 8

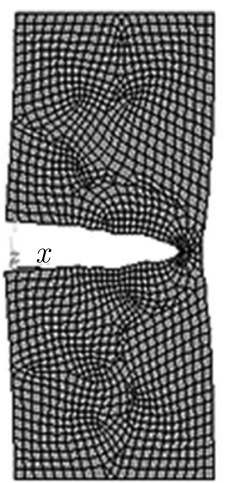

Etape 11

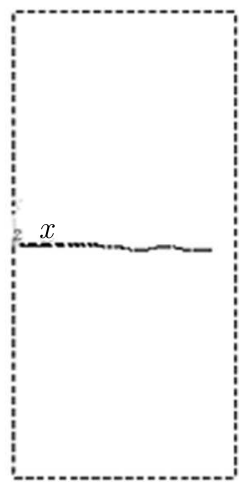

Fig. 18. Crack propagation path (NR)

(a)

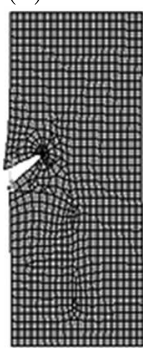

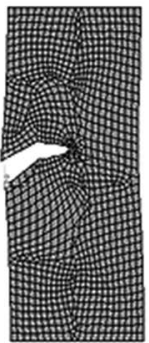
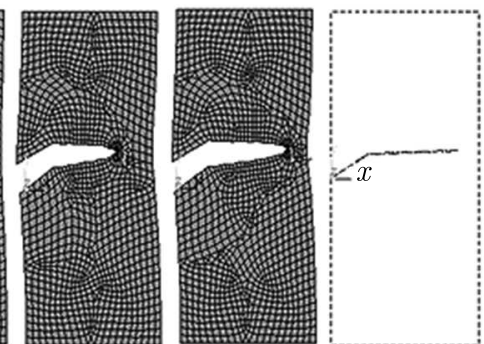

(b)

Fig. 19. Crack propagation path for an inclined crack $\left(\alpha=30^{\circ}\right)$; (a) NR, (b) SBR

\section{Conclusion}

The study has been made to analyze and simulate the crack propagation in rubber-like materials under mixed-mode loading. Using the Ansys Parametric Design Language (APDL), the strain energy density approach is investigated, at each crack increment length, the kinking angle is evaluated as a function of the Minimum Strain Energy Density (MSED) around the crack tip. The obtained results allow us to deduce the following conclusions:

- The quarter-point singular elements proposed by Barsoum (1974) are used to consider the singularity of stress and deformations fields at the crack tip. 
(a)

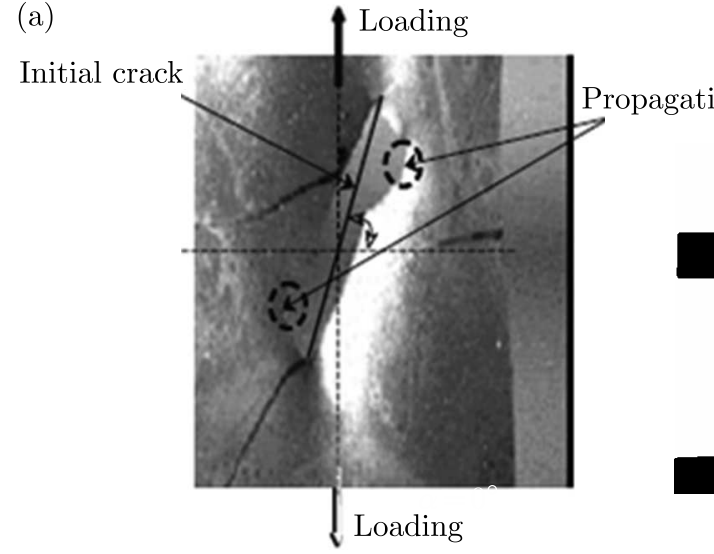

(b)
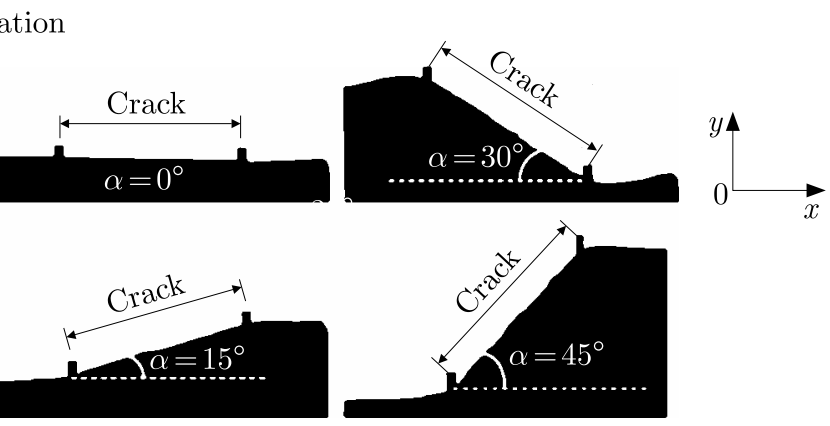

Fig. 20. Initiation and propagation of a pre-existing crack in SBR: (a) crack initiation (initial crack orientation $\alpha=45^{\circ}$ ), (b) total breaking
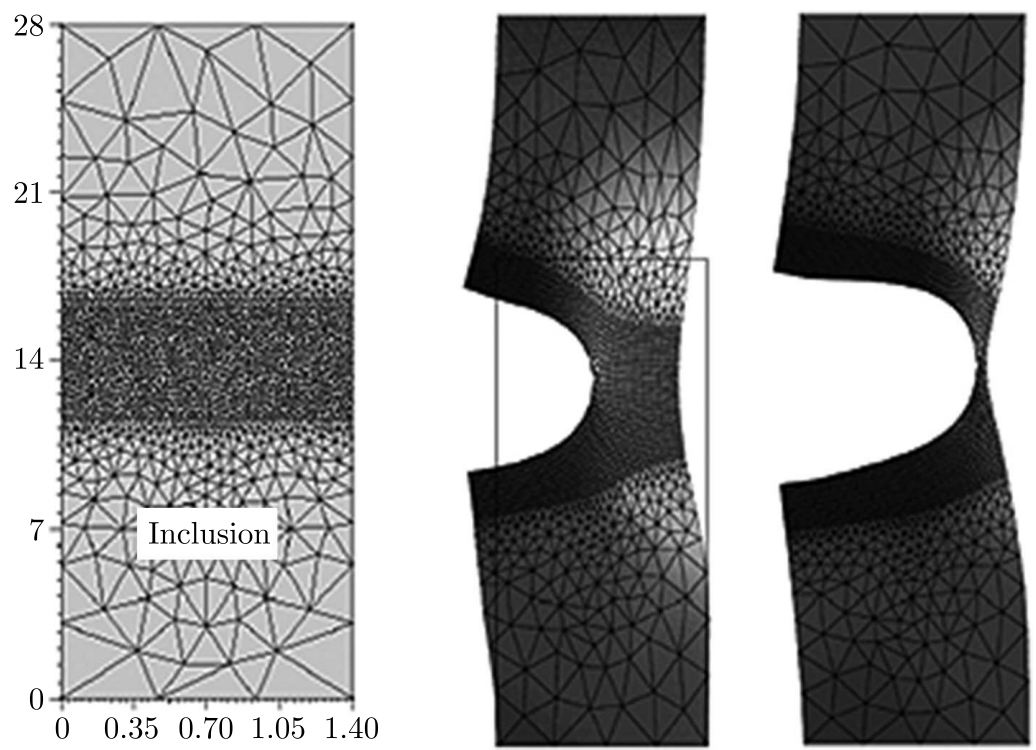

Fig. 21. Crack propagation path in the case of a rigid inclusion (Legrain, 2007)

- Out of the core region surrounding the crack tip, the minimum of SED is reached for a constant value independently of the distance $r$. We noted that the observations obtained are similar to those obtained in literature (Hamdi et al., 2006; Hamdi, 2006).

- The minimal value of SED corresponding to the direction of crack propagation is always reached in the plane perpendicular to the loading axis, independently of the initial crack orientation.

- The values of the angle of the initial crack direction and the final path of crack propagation are related to the number of the elements surrounding the crack tip.

- Consequently, the SED approach, developed in the linear elastic fracture problems, could be extended to highly non-linear deformable materials as an indicator of the crack propagation direction.

\section{References}

1. Alshonibi A., Ariffin A.K., 2006, Finite element simulation of stress intensity factors in elasticplastic crack growth, Journal of Zhejiang University - Science A, 7, 1336-1342 
2. Alshonibi A.M., Ariffin A.K., 2008. Fatigue life and crack path prediction in 2D structural components using an adaptive finite element strategy, International Journal of Mechanical and Materials Engineering, 3, 1, 97-104

3. Andrews E.H., 1974, A generalised theory of fracture mechanics, Journal of Materials Science, 9, 887-894

4. ANSYS, 2009, Inc. Programmer's Manual for Mechnical APDL, Release 12.1

5. Ayatollahi M.R., Sedighiani K., 2012, Mode I fracture initiation in limestone by strain energy density criterion, Journal of Theoretical and Applied Mechanic, 57, 14-18

6. Barsoum R.S., 1974, On the use of isoparametric finite element in linear fracture mechanics, International Journal for Numerical Methods in Engineering, 10, 25-37

7. Benouis A., Boulenouar A., Benseddiq N., Serier B., 2015, Numerical analysis of crack propagation in cement PMMA: application of SED approach, Structural Engineering and Mechanics, 55, 93-109

8. Bian L., TAheri F., 2011, A proposed maximum ratio criterion applied to mixed mode fatigue crack propagation, Materials and Design, 32, 2066-2072

9. Boulenouar A., Benouis A., Benseddiq N., 2016, Numerical modelling of crack propagation in cement PMMA: Comparison of different criteria, Materials Research Bulletin, in press

10. Boulenouar A., Benseddiq N., Mazari M., 2013a, Strain energy density prediction of crack propagation for 2D linear elastic materials, Journal of Theoretical and Applied Mechanic, 67/68, $29-37$

11. Boulenouar A., Benseddiq N., Mazari M., 2013b, Two-dimensional numerical estimation of stress intensity factors and crack propagation in linear elastic analysis, Engineering, Technology and Applied Science Research, ETASR, 3, 506-510

12. Boulenouar A., Benseddiq N., Mazari M., 2014, FE Model for linear-elastic mixed mode loading: estimation of SIFs and crack propagation, Journal of Theoretical and Applied Mechanic, 52, 373-383

13. Boulenouar A., Mazari M., 2009, Study of elastomers behavior at rupture based on the first Seth strain measures invariant, Computational Materials Science, 45, 966-971

14. CARPinteri A., 1984, Size effects in material strength due to crack growth and material nonlinearity, Journal of Theoretical and Applied Mechanic, 2, 39-45

15. Chang K.J., 1981a, Further studies of the maximum stress criterion on the angled crack problem, Engineering Fracture Mechanics, 14, 125

16. Chang K.J., 1981b, On the maximum strain criterion - a new approach to the angled crack problem, Engineering Fracture Mechanics, 14, 107-124

17. Choi D.H., Choi H.Y., Lee D., 2006, Fatigue life prediction of in-plane gusset welded joints using strain energy density factor approach, Journal of Theoretical and Applied Mechanic, 45, 108-116

18. Chow C.L., XU J., 1985, Application of the strain energy density criterion to ductile fracture, Journal of Theoretical and Applied Mechanic, 3, 185-191

19. Erdogan F., Sin G.C., 1963, On the crack extension in plates under plane loading and transverse shear, Journal of Basic Engineering, 85, 519-527

20. Fajdiga G., Ren Z., Kramar J., 2007, Comparison of virtual crack extension and strain energy density methods applied to contact surface crack growth, Engineering Fracture Mechanics, 74, $2721-2734$

21. Greensmith H.W., 1963, Rupture of rubber-X. The change in stored energy on making a small cut in a test piece held in simple extension, Journal of Applied Polymer Science, 7, 993-1002

22. Griffith A.A., 1920, The phenomenon of rupture and flow in solids, Philosophical Transactions of the Royal Society A, 221, 163-198 
23. Griffith A.A., 1924, The theory of rupture, Proceedings of 1st International Conference for Applied Mechanics, Delft, 55-63

24. Hamdi A., 2006, Critère de rupture gnralis pour les élastomères vulcanisables et thermoplastiques, Thèse de doctorat, Universit des Sciences et Technologies de Lille

25. Hamdi A., Aїт Hocine N., Naït Abdelaziz M., Benseddiq N., 2007, Fracture of elastomers under static mixed mode: the strain-energy-density factor, International Journal of Fracture, 144, $65-75$

26. Hamdi A., Nä̈т Abdelaziz M., Aït Hocine N., Benseddiq N., Heuillet P., 2006, Fracture criteria of rubber like-materials under plane stress conditions, Polymer Testing, 25, 994-1005

27. Kipp M.E., Sin G.C., 1975, The strain energy density failure criterion applied to notched elastic solids, International Journal of Solids and Structures, 11, 153-173

28. Komori K., 2005, Ductile fracture criteria for simulating shear by node separation method, Journal of Theoretical and Applied Mechanic, 43, 101-114

29. LEGRAIN G., 2007, Extension de l'approche X-FEM aux grandes transformations pour la fissuration des milieux hyperlastiques, Thèse de Doctorat, Ecole Centrale Nantes, Université de Nantes, France

30. Legrain G., Moes N., Verron E., 2005, Stress analysis around crack tips in finite strain problems using the extended Finite Element Method, International Journal for Numerical Methods in Engineering, 63, 2, 290-314

31. Maiti S.K., Smith R.A., 1983, Comparison of the criteria for mixed mode brittle fracture based on the preinstability stress-strain field, International Journal of Fracture, 23, 281-295

32. Matic P., 1985, Numerically predicting ductile material behavior from tensile specimen response, Journal of Theoretical and Applied Mechanic, 4, 13-28

33. Ogden R.W., 1984, Non Linear Elastic Deformation, Ellis Horwood Limited Publishers

34. Pegorin F., Kotousov A., Berto F., Swain M.V., Sornsuwan T., 2012, Strain energy density approach for failure evaluation of occlusal loaded ceramic tooth crowns, Journal of Theoretical and Applied Mechanic, 5, 44-50

35. Rivlin R.S., Thomas A.G., 1953, Rupture of rubber. I. Characteristic energy for tearing, Journal of Polymer Science, 10, 291-318

36. SiH G.C., 1973, Some basic problems in fracture mechanics and new concepts, Engineering Fracture Mechanics, 5, 365-377

37. SiH G.C., 1974, Strain energy-density factor applied to mixed mode crack problems, International Journal of Fracture, 10, 205

38. Sin G.C., Barthelemy B.M., 1980, Mixed mode fatigue crack growth predictions, Engineering Fracture Mechanics, 13, 439-451

39. Sih G.C., Macdonald B., 1974, Fracture mechanics applied to engineering problems - strain energy density fracture criterion, Engineering Fracture Mechanics, 6, 361-386

40. TheOcaris P.S., 1984, A higher-order approximation for the T-criterion of fracture in biaxial fields, Engineering Fracture Mechanics, 19, 975

41. Wu H.C., 1974, Dual failure criterion for plane concrete, Journal of the Engineering Mechanics Division, 100, 1167

42. YЕОн O.H., 1990, Characterization of elastic properties of carbon black filled rubber vulcanizates, Rubber Chemistry and Technology, 63, 792-805 